\title{
Subtraction of unidirectionally encoded images for suppression of heavily isotropic objects (SUSHI) for selective visualization of peripheral nerves
}

\author{
Taro Takahara • Thomas C. Kwee • Jeroen Hendrikse • \\ Marc Van Cauteren • Dow-Mu Koh • Tetsu Niwa • \\ Willem P. Th. M. Mali • Peter R. Luijten
}

Received: 1 March 2010 /Accepted: 23 April 2010/Published online: 18 May 2010

(C) The Author(s) 2010. This article is published with open access at Springerlink.com

\begin{abstract}
Introduction The aim of this study was to introduce and assess a new magnetic resonance (MR) technique for selective peripheral nerve imaging, called "subtraction of unidirectionally encoded images for suppression of heavily isotropic objects" (SUSHI).

Methods Six volunteers underwent diffusion-weighted MR neurography (DW-MRN) of the brachial plexus, and seven volunteers underwent DW-MRN of the sciatic, common peroneal, and tibial nerves at the level of the knee, at $1.5 \mathrm{~T}$. DW-MRN images with SUSHI (DW-MRN $\mathrm{SUSHI}_{\text {) and }}$ conventional DW-MRN images (DW-MRN $\mathrm{AP}_{\mathrm{P}}$ ) were displayed using a coronal maximum intensity projection and evaluated by two independent observers regarding signal suppression of lymph nodes, bone marrow, veins, and articular fluids and regarding signal intensity of nerves and ganglia, using five-point grading scales. Scores of DW$\mathrm{MRN}_{\mathrm{SUSHI}}$ were compared to those of DW-MRN $\mathrm{AP}_{\mathrm{AP}}$ using Wilcoxon tests.
\end{abstract}

Electronic supplementary material The online version of this article (doi:10.1007/s00234-010-0713-6) contains supplementary material, which is available to authorized users.

T. Takahara $(\bowtie) \cdot$ T. C. Kwee $\cdot$ J. Hendrikse $\cdot$ T. Niwa $\cdot$

W. P. T. M. Mali $\cdot$ P. R. Luijten

Department of Radiology, University Medical Center Utrecht,

Heidelberglaan 100,

3508GX Utrecht, The Netherlands

e-mail: tarorin@rr.iij4u.or.jp

M. Van Cauteren

Philips Healthcare, Asia Pacific,

Tokyo, Japan

D.-M. Koh

Department of Radiology, Royal Marsden Hospital,

Sutton, UK
Results Suppression of lymph nodes around the brachial plexus and suppression of articular fluids at the level of the

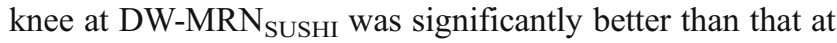
$\mathrm{DW}_{-M R N_{\mathrm{AP}}}(P<0.05)$. However, overall signal intensity of brachial plexus nerves and ganglia at DW-MRN $\mathrm{MUSHI}_{\mathrm{SH}}$ was significantly lower than that at DW-MRN $\mathrm{AP}_{\mathrm{P}}(P<0.05)$. On the other hand, signal intensity of the sciatic, common peroneal, and tibial nerves at the level of the knee at DWMRN $_{\text {SUSHI }}$ was judged as significantly better than that at DW-MRN $_{\mathrm{AP}}(P<0.05)$.

Conclusion The SUSHI technique allows more selective visualization of the sciatic, common peroneal, and tibial nerves at the level of the knee but is less useful for brachial plexus imaging because signal intensity of the brachial plexus nerves and ganglia can considerably be decreased.

Keywords Diffusion-weighted MR neurography - SUSHI · Peripheral nerves · Brachial plexus · Diffusion-weighed imaging
Abbreviations
DW-MRN Diffusion-weighted MR neurography
DW-
DW-MRN images with SUSHI
$\mathrm{MRN}_{\mathrm{SUSHI}}$
DW-
DW-MRN created by the axial source dataset
$\mathrm{MRN}_{\mathrm{AP}}$ acquired using MPGs in the anterior-poste- rior direction only
DW- $\quad$ DW-MRN created by the axial source dataset
$\mathrm{MRN}_{\mathrm{SI}}$ acquired using MPGs in the superior-inferior direction only
DTI Diffusion tensor imaging
EPI Echo planar imaging
FOV Field of view
MIP 


$\begin{array}{ll}\text { MPG } & \text { Motion probing gradient } \\ \text { MR } & \text { Magnetic resonance } \\ \text { STIR } & \text { Short tau inversion recovery } \\ \text { SUSHI } & \begin{array}{l}\text { Subtraction of unidirectional encoded } \\ \text { images for suppression of heavily isotropic } \\ \text { objects }\end{array}\end{array}$

\section{Introduction}

Magnetic resonance (MR) imaging provides high-resolution and excellent soft tissue contrast, making it a very useful modality to evaluate peripheral nerves [1-4]. However, MR sequences that are commonly used in clinical practice such as T1- and (fat-suppressed) T2-weighted imaging are not capable of visualizing peripheral nerves over a long trajectory because of the close anatomic relationship between peripheral nerves and other structures with similar signal intensities on these sequences (e.g., surrounding muscle bundles exhibit low signal intensity on T1-weighted imaging and vascular structures exhibit high signal intensity on T2-weighted imaging) [1-4]. Consequently, image interpretation can be time-consuming, and lesions may be missed due to the fact that many slices in different planes have to be evaluated. In addition, although it has been reported that it is possible to visualize peripheral nerves over a long trajectory if T1-weighted 3D datasets are acquired and displayed using curved multiplanar reconstructions [5], such an approach requires time-consuming postprocessing steps. To overcome the drawbacks of conventional MR sequences, diffusion-weighted MR neurography (DW-MRN) was recently introduced [6]. In DWMRN, peripheral nerves are highlighted and can be visualized over long trajectories, while most background body signals are suppressed thanks to the use of heavy diffusion-weighting and fat suppression [6]. Note that DWMRN is different from diffusion tensor imaging (DTI) in that it is just imaging of nerves and does not track the anisotropy of nerves [6]. Extending this concept to the entire body even allows whole-body MR neurographic imaging [7]. However, selective visualization of peripheral nerves has not been achieved yet because several normal structures, such as bone marrow and lymph nodes (e.g., around the brachial plexus) and veins with slow blood flow and articular fluids (e.g., around the common peroneal and tibial nerves at the level of the knee), maintain a high signal intensity at DW-MRN $[6,7]$. The purpose of this study was to introduce and assess the utility of the "subtraction of unidirectionally encoded images for suppression of heavily isotropic objects" (SUSHI) technique for more selective visualization of peripheral nerves at DW-MRN.

\section{Materials and methods}

Study participants

This study was approved by the local institutional review board, and written informed consent was obtained from all participants. Six healthy volunteers (three men and three women; mean age, 27 years; age range, 21-48 years) underwent DW-MRN of the brachial plexus, and seven other healthy volunteers (five men and two women; mean age, 29 years; age range, 24-47 years) underwent DWMRN of the sciatic, common peroneal, and tibial nerves at the level of the knee. Exclusion criteria were general contraindications to MR imaging, such as implanted pacemaker and claustrophobia.

\section{SUSHI technique}

It has been shown that diffusion is anisotropic in the human nervous system, including the peripheral nerves [8-11]. In other words, diffusion is more impeded perpendicular to the course of the nerves, while the highest diffusivity can be expected parallel to the course of the nerves. For this reason, the highest signal intensity of the peripheral nerves can be obtained by applying only one pair of motion probing gradients (MPGs) perpendicular to the course of the nerves, while signal intensity of the peripheral nerves is the lowest when applying MPGs parallel to the course of the nerves; this was recently confirmed in studies on DWMRN of the sacral plexus [12] and sciatic nerve [13]. On the other hand, many structures that surround the nerves, including lymph nodes, bone marrow, veins with slow blood flow, and articular fluids, always exhibit a relatively high signal intensity, regardless of the applied direction of MPGs [12]. Based on these facts [8-13], it can be hypothesized that selective visualization of peripheral nerves can be achieved by subtracting one DW-MRN dataset obtained with only one pair of MPGs parallel to the course of the nerves from another DW-MRN dataset obtained with a pair of MPGs perpendicular to the course of the nerves. This technique was called SUSHI.

\section{MR imaging}

Six volunteers underwent DW-MRN of the brachial plexus, and seven other volunteers underwent DW-MRN of the sciatic, common peroneal, and tibial nerves at the level of the knee using a 1.5-T MR system (Achieva, Philips Healthcare, Best, The Netherlands) with either a fourelement $(n=1)$, an eight-element $(n=7)$, or an 18-element $(n=5)$ phased-array surface coil for signal reception. Imaging parameters for DW-MRN of the brachial plexus were as follows: single-shot short tau inversion recovery- 
echo planar imaging (EPI), axial plane, repetition time (TR)/ echo time (TE)/inversion time of 4,665:85:180 ms, field of view (FOV) of $250 \times 400 \mathrm{~mm}^{2}$, acquisition matrix of $160 \times$ 98 , slice thickness/overlap of 4:0 mm, number of slices of 60 , half scan factor (half Fourier) of 0.6, EPI factor (echo train length) of 53, parallel acquisition (sensitivity encoding) factor of 2, number of directions of MPGs of 2 (anteriorposterior and superior-inferior), $b$ value of $900 \mathrm{~s} / \mathrm{mm}^{2}$, and number of excitations (NEX) of 8 . Both directions of MPGs were applied in one scan, and total acquisition time was 2 min $48 \mathrm{~s}$. Imaging parameters for DW-MRN of the sciatic, common peroneal, and tibial nerves at the level of the knee were similar, except for a TR of $9,167 \mathrm{~ms}$, a TE of $82 \mathrm{~ms}$, FOV of $174 \times 220 \mathrm{~mm}^{2}$, acquisition matrix of $96 \times 72$, slice thickness/overlap of 2.5:0 mm, EPI factor of 39, NEX of 32, and total acquisition time of $5 \mathrm{~min} 11 \mathrm{~s}$.

\section{Postprocessing}

DW-MRN images with SUSHI (DW-MRN SUSHI $_{\text {) were }}$ created by subtracting the axial source dataset acquired using MPGs in the superior-inferior direction only (DW$\mathrm{MRN}_{\mathrm{SI}}$ ) from the axial source dataset acquired using MPGs in the anterior-posterior direction only $\left(\mathrm{DW}-\mathrm{MRN}_{\mathrm{AP}}\right)$ by means of software implemented in the standard console of the MR system. Subsequently, axial DW-MRN $N_{\text {SUSHI }}$ and axial $\mathrm{DW}-\mathrm{MRN}_{\mathrm{AP}}$ images were displayed in the coronal plane using a maximum intensity projection (MIP). Grayscale inversion was applied.

\section{Image analysis}

Coronal MIP DW-MRN $\mathrm{SUSHI}_{\text {and }}$ aW-MRN $\mathrm{AP}_{\mathrm{AP}}$ images were independently evaluated by two radiologists (T.T. and T.N., with 20 and 12 years of experience in MR imaging interpretation, respectively). Any information regarding the applied MR sequence was removed from all images, and images were evaluated in a random order.

First, signal suppression of lymph nodes and bone marrow (for DW-MRN of the brachial plexus) and signal suppression of veins and articular fluids (for DW-MRN of the sciatic, common peroneal, and tibial nerves at the level of the knee) were assessed separately using a fivepoint grading scale $(1=$ very poor suppression, structure of interest [i.e., lymph nodes, bone marrow, veins, or articular fluids] shows very high signal intensity relative to that of surrounding fat; 2 = poor suppression, structure of interest shows high signal intensity relative to that of surrounding fat; 3 = moderate suppression, structure of interest shows moderately high signal intensity relative to that of surrounding fat; 4 = good suppression, structure of interest shows only slightly higher signal than surrounding fat; 5 = very good suppression, structure of interest
Table 1 Comparison of scores between DW-MRN $N_{\text {SUSHI }}$ and DW$\mathrm{MRN}_{\mathrm{AP}}$ of the brachial plexus regarding signal suppression of lymph nodes and bone marrow, for both observers.

\begin{tabular}{lcccc}
\hline Structure & \multirow{2}{*}{ Observer } & \multicolumn{2}{l}{ Median score (range) } & \multirow{2}{*}{$\begin{array}{l}P \\
\text { value }^{\mathrm{a}}\end{array}$} \\
\cline { 3 - 4 } & & DW-MRN $_{\text {SUSHI }}$ & DW-MRN $_{\mathrm{AP}}$ & \\
\hline $\begin{array}{l}\text { Lymph } \\
\text { nodes }\end{array}$ & 1 & $5(4-5)$ & $2(1-3)$ & 0.020 \\
Bone & 2 & $4(3-5)$ & $1(1)$ & 0.026 \\
marrow & 1 & $5(4-5)$ & $3(1-5)$ & 0.059 \\
& 2 & $5(\mathrm{NA})$ & $3.5(2-5)$ & 0.066 \\
\hline
\end{tabular}

$N A$ not applicable (the same score was assigned to all images in this group)

${ }^{\mathrm{a}}$ Wilcoxon test

shows no or a very low signal intensity, equal to that of surrounding fat).

Second, signal intensity of the nerves (for both DWMRN of the brachial plexus and DW-MRN of the sciatic, common peroneal, and tibial nerves at the level of the knee) and signal intensity of the ganglia (for DW-MRN of the brachial plexus only) were assessed separately using another five-point grading scale $(1=$ very poor signal intensity, structure of interest [i.e., nerves or ganglia] shows no or a very low signal intensity, equal to that of surrounding fat; $2=$ poor signal intensity; structure of interest shows only slightly higher signal intensity relative to that of surrounding fat; $3=$ moderate signal intensity, structure of interest shows moderately high signal intensity relative to that of surrounding fat; 4 = good signal intensity, structure of interest shows high signal intensity relative to that of surrounding fat; $5=$ very good signal intensity, structure of interest shows very high signal intensity relative to that of surrounding fat).

\section{Statistical analysis}

Because of the use of data on an ordinal scale, scores were expressed as medians with ranges and compared using

Table 2 Comparison of scores between DW-MRN $\mathrm{SUSHI}_{\text {and }}$ DW$\mathrm{MRN}_{\mathrm{AP}}$ of the brachial plexus regarding signal intensity of nerves and ganglia, for both observers.

\begin{tabular}{lcccc}
\hline Structure & Observer & \multicolumn{2}{l}{ Median score (range) } & \multirow{2}{*}{$P$ value $^{\mathrm{a}}$} \\
\cline { 3 - 4 } & & DW-MRN $_{\text {SUSHI }}$ & DW-MRN $_{\mathrm{AP}}$ & \\
\hline Nerves & 1 & 3 (NA) & $4.5(4-5)$ & 0.024 \\
& 2 & $3(\mathrm{NA})$ & $4.5(4-5)$ & 0.024 \\
Ganglia & 1 & 1 (NA) & $4(3-5)$ & 0.026 \\
& 2 & $1(1-2)$ & $5(4-5)$ & 0.023 \\
\hline
\end{tabular}

$N A$ not applicable (i.e., the same score was assigned to all images in this group)

${ }^{\mathrm{a}}$ Wilcoxon test 

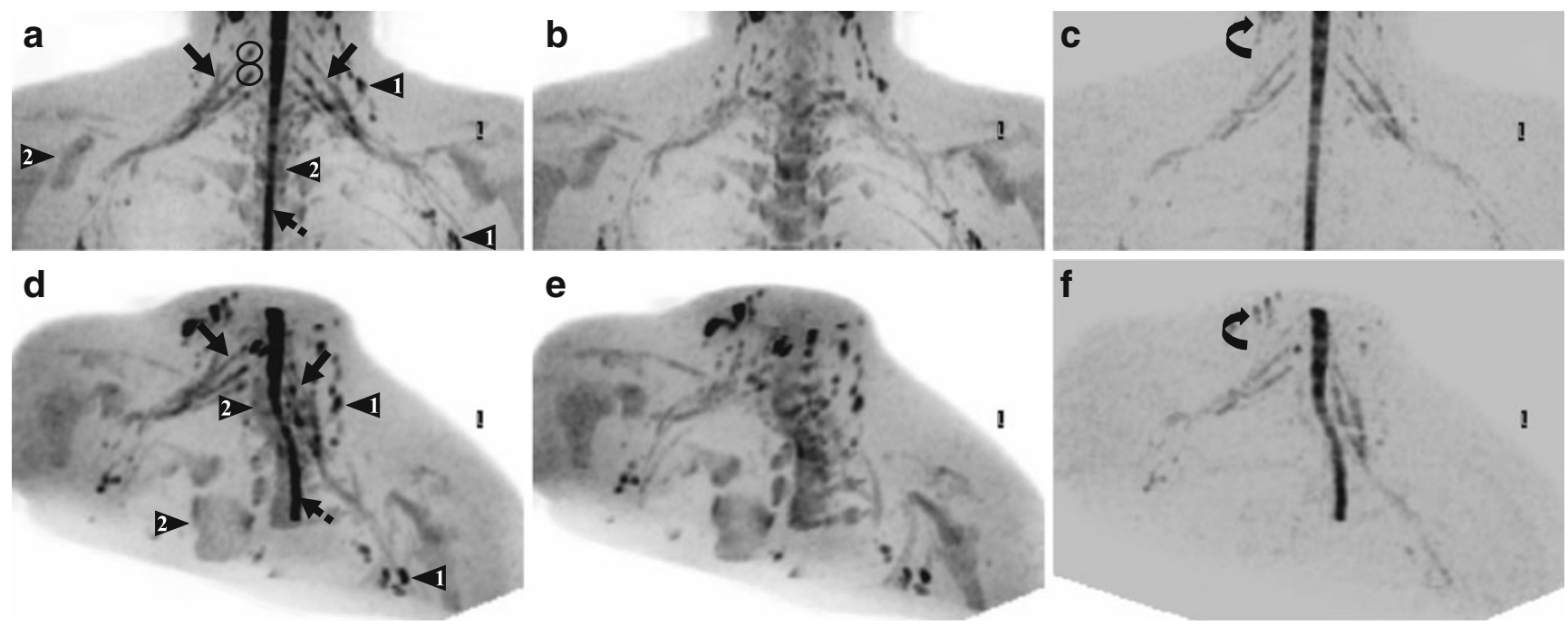

Fig. 1 Comparison of DW-MRN $\mathrm{AP}_{\mathrm{A}}(\mathbf{a}, \mathbf{d}), \mathrm{DW}-\mathrm{MRN}_{\mathrm{SI}}(\mathbf{b}, \mathbf{e})$, and $\mathrm{DW}_{-M R N_{\mathrm{SUSHI}}}\left(\mathrm{DW}-\mathrm{MRN}_{\mathrm{AP}}\right.$ minus DW-MRN $\left.\mathrm{SI} ; \mathbf{c}, \mathbf{f}\right)$ of the brachial plexus in a 21-year-old healthy female, displayed using coronal MIPs $(\mathbf{a}-\mathbf{c})$ and rotated MIPs. Images were acquired using a four-element phased-array surface coil. DW-MRN $\mathrm{AP}_{\mathrm{AP}}(\mathbf{a}, \mathbf{d})$ shows the nerves of the brachial plexus (continuous arrows), ganglia (encircled), spinal cord (dashed arrows), lymph nodes (arrowheads \#1), and bone marrow (arrowheads \#2), with some degree of overprojection between the brachial plexus and several surrounding structures. In DW-MRN $\mathrm{SI}_{\mathrm{SI}}(\mathbf{b}$, e), nerves of the brachial plexus are not or poorly visualized due to their diffusion anisotropy while structures of isotropic diffusivity (including lymph nodes and bone marrow) maintain their high signal intensity. In DW-MRN $\mathrm{SUSHI}_{\mathrm{S}}(\mathbf{c}, \mathbf{f})$ nerves of the brachial plexus are selectively visualized because of efficient subtraction of surrounding structures of isotropic diffusivity. However, note some degree of signal loss of the brachial plexus. Furthermore, note that ganglia are also suppressed due to their isotropic diffusivity and that slight residual signal from insufficiently suppressed lymph nodes can be seen (curved arrows), probably due to motion of the subject during image acquisition or due to image distortion originated from eddy current, resulting in imperfect subtraction nonparametric tests. Median scores regarding signal suppression of lymph nodes and bone marrow (for DW-MRN of the brachial plexus) and that of veins and articular fluids (for DW-MRN of the sciatic, common peroneal, and tibial nerves at the level of the knee) were calculated, for both observers separately. Furthermore, median scores regarding signal intensity of the nerves (for both DWMRN of the brachial plexus and DW-MRN of the sciatic, common peroneal, and tibial nerves at the level of the knee) and that of the ganglia (for DW-MRN of the brachial plexus only) were calculated, for both observers separately. Scores of DW-MRN ${ }_{\text {SUSHI }}$ were compared to those of DW-MRN ${ }_{\mathrm{AP}}$ using Wilcoxon tests. Differences were considered significant when $P$ values were less than 0.05 . Statistical analyses were executed using Statistical Package for the Social Sciences (SPSS) software version 16.0 (SPSS, Chicago, IL, USA).

\section{Results}

\section{Brachial plexus}

Results are displayed in Tables 1 and 2. DW-MRN $\mathrm{SUSHI}_{\mathrm{S}}$ yielded a significantly better suppression of lymph nodes than DW-MRN $\mathrm{AP}_{\mathrm{AP}}(P<0.05$ for both observers $)$, and there was a trend toward a better suppression of bone marrow at DW-MRN ${ }_{\text {SUSHI }}$ compared to that at DW-MRN $\mathrm{AP}_{\mathrm{AP}}(P=0.059$ and $P=0.066$ for observers 1 and 2, respectively). On the other hand, overall signal intensity of nerves at DWMRN $_{\text {SUSHI }}$ was moderate and significantly lower than that at DW-MRN $\mathrm{AP}_{\mathrm{AP}}(P<0.05$ for both observers). Furthermore, overall signal intensity of ganglia at DW-MRN $\mathrm{SUSHI}_{\mathrm{SH}}$ was poor to very poor and significantly lower than that at DW$\mathrm{MRN}_{\mathrm{AP}}(P<0.05$ for both observers). Representative examples of DW-MRN $\mathrm{SUSHI}_{\mathrm{SU}}$ compared to DW-MRN $\mathrm{AP}_{\mathrm{AP}}$ of the brachial plexus are shown in Figs. 1 and 2.

Common peroneal and tibial nerves at the level of the knee

Results are displayed in Tables 3 and 4. DW-MRN ${ }_{\text {SUSHI }}$ yielded a significantly better suppression of articular fluids than DW-MRN $\mathrm{AP}_{\mathrm{AP}}(P<0.05$ for both observers). Suppression of veins, however, was not significantly different between the two techniques ( $P>0.1$ for both observers). Overall signal intensity of nerves at DW-MRN $\mathrm{SuSH}_{\mathrm{SH}}$ was judged as good to very good, which was significantly better than that at DW-MRN $\mathrm{AP}_{\mathrm{P}}(P<0.05$ for both observers $)$. A representative example of DW-MRN $\mathrm{SUSHI}_{\mathrm{SU}}$ compared to DW-MRN $\mathrm{AP}_{\mathrm{P}}$ is shown in Fig. 3 and Movie clip 1. 

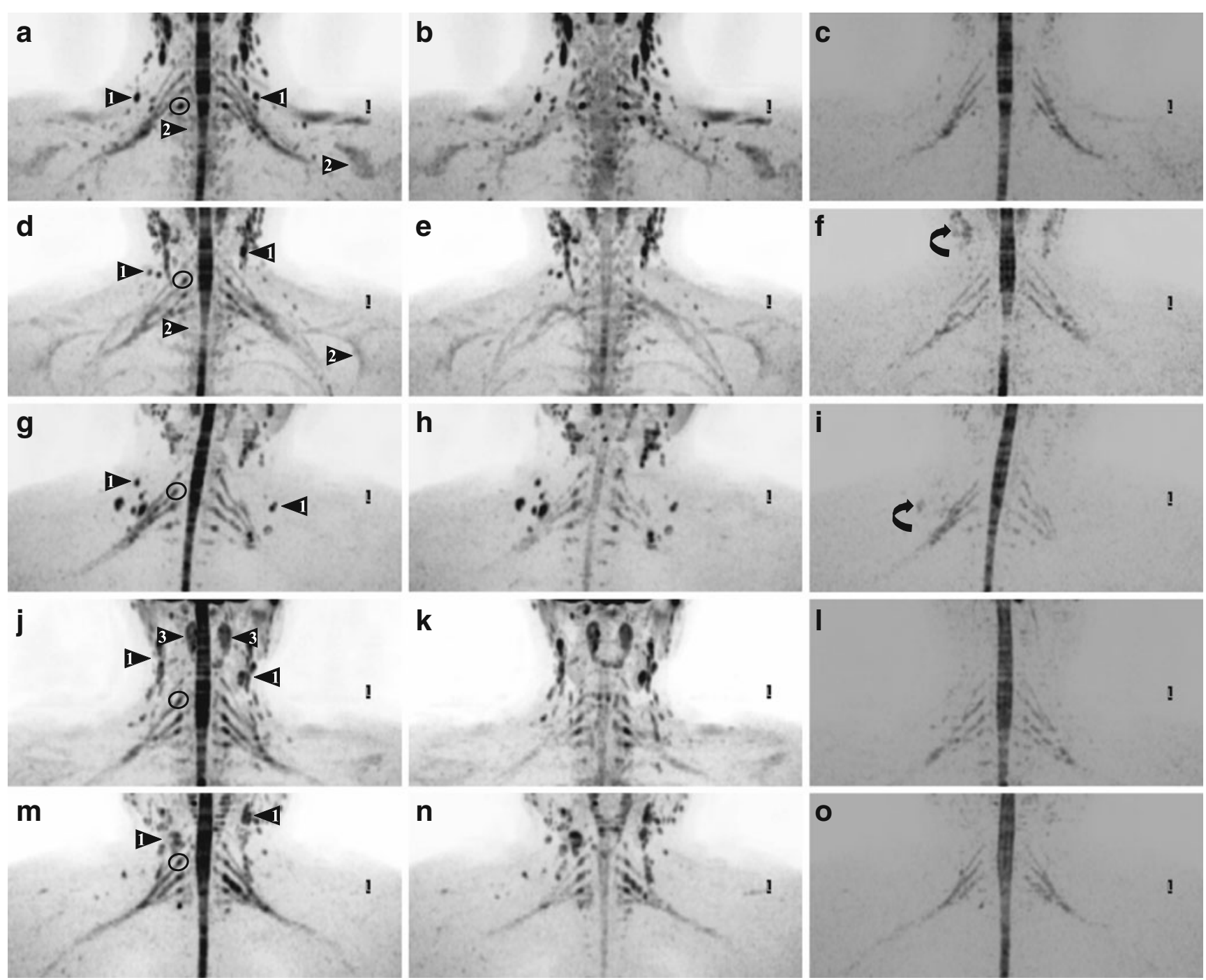

Fig. 2 Comparison of DW-MRN $\mathrm{AP}(\mathbf{a}, \mathbf{d}, \mathbf{g}, \mathbf{j}, \mathbf{m}), \mathrm{DW}-\mathrm{MRN}_{\mathrm{SI}}(\mathbf{b}, \mathbf{e}$, $\mathbf{h}, \mathbf{k}, \mathbf{n})$, and DW-MRN $\mathrm{SUSHI}_{\mathrm{SW}}\left(\mathrm{DW}-\mathrm{MRN}_{\mathrm{AP}}\right.$ minus DW-MRN $\mathrm{SI}_{\mathrm{SI}} ; \mathbf{c}, \mathbf{f}, \mathbf{i}$, l, o) of the brachial plexus in five consecutive volunteers (volunteer 1 $(\mathbf{a}-\mathbf{c})$, volunteer $2(\mathbf{d}-\mathbf{f})$, volunteer $3(\mathbf{g}-\mathbf{i})$, volunteer $4(\mathbf{j}-\mathbf{l})$, volunteer $5(\mathbf{m}-\mathbf{0}))$, displayed using coronal MIPs. Images were acquired using a dedicated neurovascular 18-element phased-array surface coil. DW$\mathrm{MRN}_{\mathrm{AP}}(\mathbf{a}, \mathbf{d}, \mathbf{g}, \mathbf{j}, \mathbf{m})$ shows the brachial plexus, lymph nodes (arrowheads \#1), bone marrow (arrowheads \#2), and tonsils (arrowheads \#3), with some degree of overprojection between the brachial

\section{Discussion}

DW-MRN is a recently introduced technique for peripheral nerve imaging $[6,7]$. A major advantage of this technique over conventional (T1- and [fat-suppressed] T2-weighted) MR sequences is its capability to visualize nerves over long trajectories because of excellent suppression of background structures such as fat, muscles, and vascular structures $[6,7]$. Nevertheless, although many unwanted structures are suppressed at DW-MRN, several normal structures that have a relatively long $\mathrm{T} 2$ value and an impeded diffusion plexus and several surrounding structures. In DW-MRN $N_{\mathrm{SI}}(\mathbf{b}, \mathbf{e}, \mathbf{h}, \mathbf{k}$, n), nerves of the brachial plexus are not or poorly visualized due to their diffusion anisotropy while other structures maintain their high signal intensity. In DW-MRN $\mathrm{SUSHI}_{\mathrm{SU}}(\mathbf{c}, \mathbf{f}, \mathbf{i}, \mathbf{l}, \mathbf{o})$ nerves of the brachial plexus are selectively visualized. Again, note some degree of signal loss of the brachial plexus that ganglia (encircled in $\mathbf{a}, \mathbf{d}, \mathbf{g}, \mathbf{j}, \mathbf{m}$ ) are also suppressed due to their isotropic diffusivity and that slight residual signal from insufficiently suppressed lymph nodes can be seen in some cases (curved arrows)

(among which lymph nodes, bone marrow, veins with slow blood flow, and articular fluids) maintain a high signal intensity $[6,7]$. The presence of these structures in the near proximity of the nerves and superimposition of these structures on the nerves on projection images may hinder the evaluation of the nerves.

Because of the diffusion anisotropy of the human nervous system [8-11], peripheral nerves are best visualized when applying only one pair of MPGs perpendicular to the course of the nerves, while nerves are not well visualized when applying MPGs parallel to the course of 
Table 3 Comparison of scores between DW-MRN ${ }_{\text {SUSHI }}$ and DW$\mathrm{MRN}_{\mathrm{AP}}$ of the common peroneal and tibial nerves at the level of the knee regarding suppression suppression of veins and articular fluids, for both observers.

\begin{tabular}{lclll}
\hline Structure & Observer & \multicolumn{2}{l}{ Median score (range) } & \multirow{2}{*}{$P$ value $^{\mathrm{a}}$} \\
\cline { 3 - 4 } & & $\begin{array}{l}\mathrm{DW}- \\
\mathrm{MRN}_{\text {SUSHI }}\end{array}$ & $\begin{array}{l}\text { DW- } \\
\mathrm{MRN}_{\mathrm{AP}}\end{array}$ \\
\hline Veins & 1 & $2(1-3)$ & $2(1-3)$ & 0.157 \\
& 2 & $2(1-4)$ & $1(1-3)$ & 0.102 \\
Articular fluids & 1 & $4(3-5)$ & $1(1-2)$ & 0.016 \\
& 2 & $4(3-5)$ & 1 (NA) & 0.017
\end{tabular}

$N A$ not applicable (i.e., the same score was assigned to all images in this group)

${ }^{\mathrm{a}}$ Wilcoxon test

the nerves $[12,13]$. On the other hand, applying different directions of MPGs does not affect signal intensity of surrounding normal structures, such as lymph nodes and bone marrow, because these structures exhibit an isotropic diffusivity. The proposed SUSHI technique exploits the difference in diffusion (an)isotropy between the nerves and surrounding structures and provides an "anisotropy-enhanced" image. The results of the present study show that DW-MRN ${ }_{\text {SUSHI }}$ provides a significantly better suppression of lymph nodes and articular fluids surrounding the nerves than conventional (unsubtracted) DW-MRN $\mathrm{AP}_{\mathrm{AP}}$. Although there was only a trend toward better suppression of bone marrow surrounding the brachial plexus at DW-MRN ${ }_{\mathrm{SUSHI}}$ compared to that at DW-MRN $\mathrm{AP}_{\mathrm{AP}}$, this can be explained by the fact that not all volunteers had high signal intensity bone marrow at $\mathrm{DW}-\mathrm{MRN}_{\mathrm{AP}}$ (note that this condition is probably related to the amount of hematopoietic bone marrow [14]). On the other hand, suppression of veins at the level of the knee at DW-MRN $\mathrm{SUSH}_{\text {SUI }}$ was not significantly better than that at DW-MRN $\mathrm{AP}_{\mathrm{AP}}$

Although the proposed SUSHI technique can be regarded as an important step forward toward selective peripheral nerve imaging, it has several disadvantages.

Table 4 Comparison of scores between DW-MRN ${ }_{\mathrm{SUSHI}}$ and DW$\mathrm{MRN}_{\mathrm{AP}}$ of the common peroneal and tibial nerves at the level of the knee regarding signal intensity of the nerves, for both observers.

\begin{tabular}{lcccc}
\hline Structure & \multirow{2}{*}{ Observer } & \multicolumn{2}{l}{ Median score (range) } & \multirow{2}{*}{$P$ value $^{\mathrm{a}}$} \\
\cline { 3 - 4 } & & $\mathrm{DW}-\mathrm{MRN}_{\mathrm{SUSHI}}$ & $\mathrm{DW}-\mathrm{MRN}_{\mathrm{AP}}$ & \\
\hline Nerves & 1 & $4(4-5)$ & $3(2-4)$ & 0.015 \\
& 2 & $5(\mathrm{NA})$ & $4(3-5)$ & 0.038
\end{tabular}

$N A$ not applicable (i.e., the same score was assigned to all images in this group)

${ }^{\mathrm{a}}$ Wilcoxon test
First, inherent signal loss of the nerves occurs because of the use of a subtraction method. Nevertheless, overall signal intensity of the brachial plexus nerves at DW$\mathrm{MRN}_{\text {SUSHI }}$ was still graded as "moderate" and that of the sciatic, common peroneal, and tibial nerves at the level of the knee was even graded as "good" to "very good". Furthermore, signal may be improved by increasing the number of excitations. A second drawback of the SUSHI method is that it does not effectively visualize nerves that have a course that is not parallel to the direction of the second pair of MPGs. In the present study, signal intensity of brachial plexus nerves at DW-MRN $\mathrm{SUSHI}_{\mathrm{S}}$ was moderate and significantly lower than that at DW-MRN $\mathrm{AP}_{\mathrm{AP}}$. Conversely, signal intensity of the common peroneal and tibial nerves at the level of the knee at DW-MRN $\mathrm{SUSHI}_{\mathrm{S}}$ was good to very good and significantly better than that at DW$\mathrm{MRN}_{\mathrm{AP}}$. This discrepancy can be explained by differences in signal intensity of the nerves at DW-MRN $\mathrm{SI}_{\mathrm{SI}}$ that should work as "subtraction mask". Since the common peroneal and tibial nerves at the level of the knee run superiorinferiorly, they do not show any signal at DW-MRN $\mathrm{SI}_{\mathrm{S}}$, resulting in a good visualization of these nerves at DW$\mathrm{MRN}_{\mathrm{SUSHI}}$ (note that nerve signal is well maintained at DW-MRN $\mathrm{AP}_{\mathrm{AP}}$ ). Conversely, since the brachial plexus courses under an angle of approximately $45^{\circ}$ to the superior-inferior direction, it's suppression is not perfect at DW-MRN $\mathrm{SI}_{\mathrm{SI}}$, which causes a subtraction error at DW$\mathrm{MRN}_{\mathrm{SUSHI}}$ (note that the maintenance of nerve signal is moderate at $\mathrm{DW}-\mathrm{MRN}_{\mathrm{AP}}$ ). Instead of applying MPGs in only two different directions, this problem may be solved by applying a higher number of directions of MPGs that can be used for subtraction [13]. However, such an approach will increase scan time. Third, imperfect subtraction between the DW-MRN dataset acquired with MPGs perpendicular to the nerves from the DW-MRN dataset with MPGs parallel to the course of the nerves may occur in case of patient motion and/or image distortion due to the use of single-shot EPI. This, in turn, will lead to imperfect suppression of objects with an isotropic diffusivity. The development of a distortion correction technique may, in part, solve this problem. A fourth disadvantage is that brachial plexus ganglia were almost completely suppressed at DW-MRN $\mathrm{NUSH}_{\mathrm{SU}}$, probably because of the isotropic diffusivity of these structures or because of their left-right orientation. However, this problem can be solved when interpreting DW-MRN $\mathrm{N}_{\mathrm{SUSHI}}$ along with conventional (unsubtracted) DW-MRN since ganglia are not suppressed using the latter. Of note, when acquiring DW-MRN with two directions of MPGs, both a high-quality conventional (unsubtracted) DW-MRN dataset and a SUSHI (subtracted) DW-MRN dataset (enabling selective imaging of peripheral nerves) can be obtained in a clinically acceptable examination time. This is well illustrated by the fact that total scan 

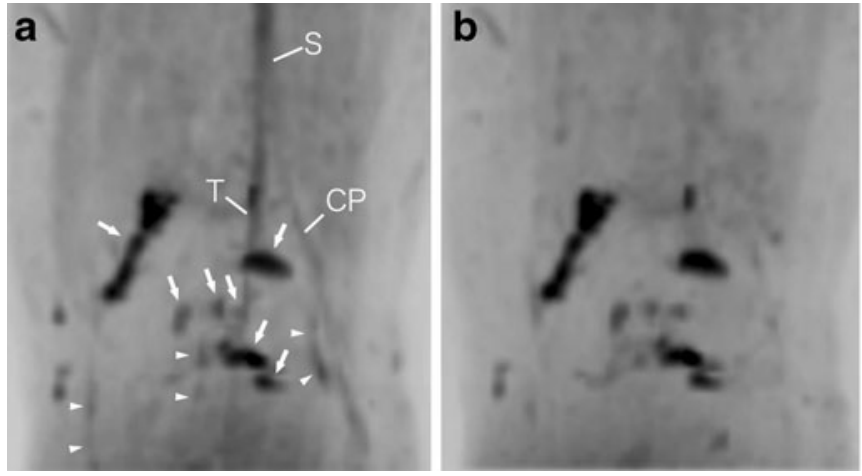

Fig. 3 Comparison of DW-MRN $\mathrm{DP}_{\mathrm{AP}}$ (a), DW-MRN $\mathrm{DI}_{\mathrm{SI}}$ (b), DW$\mathrm{MRN}_{\mathrm{SUSHI}}\left(\mathrm{DW}-\mathrm{MRN}_{\mathrm{AP}}\right.$ minus DW-MRN $\mathrm{SI} ; \mathbf{c}$ ), and normal diffusion-weighted imaging $(D W I)$ obtained with MPGs in three orthogonal axes (d) of the sciatic, common peroneal, and tibial nerves at the level of the knee in a 35-year-old male. At DW-MRN $\mathrm{AP}_{\mathrm{A}}(\mathbf{a})$, the sciatic $(S)$, tibial $(T)$, and common peroneal $(C P)$ nerves are visualized. However, articular fluids exhibit very high signal intensity (arrows) and sometimes superimpose on the nerves, which deteriorates overall image quality. Veins are also displayed (arrowheads). At
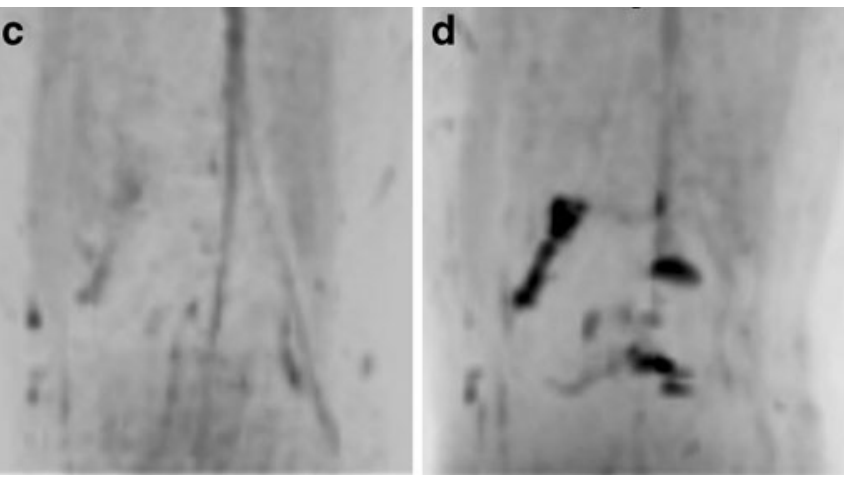

DW-MRN $\mathrm{SI}_{\mathrm{S}}(\mathbf{b})$, the nerves are almost completely suppressed because the MPGs are placed parallel to the course of the nerves. At DWMRN $_{\text {SUSHI }}(\mathbf{c})$, the nerves are well visualized thanks to elimination of signal from articular fluids, although most signals from veins are still visible. Note that normal DWI obtained with MPGs in three orthogonal axes (d) using the same scan time as DW-MRN $\mathrm{AP}_{\mathrm{AP}}$ shows poor visualization of the nerves compared to a due to the diffusion anisotropy of nerves, while visualization of articular fluids and veins is similar to that in a due to the diffusion isotropy of these structures time for either DW-MRN of the brachial plexus or DWMRN of the sciatic, common peroneal, and tibial nerves at the level of the knee was less than 6 min. Combined with conventional DW-MRN [6, 7], the proposed SUSHI technique has the potential to improve diagnosing peripheral nerve disease, optimize lesion localization, and allow a faster and more straightforward evaluation of the extent of disease than electrophysiological studies and conventional (T1- and [fat-suppressed] T2-weighted) MR sequences [14]. Importantly, although acquired DW-MRN datasets (without and with SUSHI) can be postprocessed to create visually appealing MIP images for review with referring physicians, source data should always be carefully reviewed as they provide the maximum diagnostic information.

It may be argued that a sophisticated MIP postprocessing procedure, called soap-bubble MIP, may be used instead of the SUSHI technique to eliminate unwanted normal structures surrounding the nerves in DW-MRN [6]. However, this approach requires extra postprocessing time, and it may not be successful when nerves are closely encompassed by surrounding structures. Other postprocessing techniques have been described that may provide nice depictions of the peripheral nerves $[5,15]$. However, a clear advantage of the proposed SUSHI technique over these other techniques $[5,6,15]$ is that the former one does not require any extensive postprocessing time and may be easier to implement in clinical practice. Furthermore, it should be acknowledged that only healthy volunteers and no pathologic cases were included in this study. Since the SUSHI technique reduces nerve conspicuity, it is possible that this may also reduce detectability of diffusion abnormalities in pathologic nerves. In some types of nerve pathology such as traumatic nerve injury, lesions may lose their anisotropy and the SUSHI technique would actually remove the structures in this case. Furthermore, pathologic nerve compression may result in deviation of the course of the nerves as a result of which the MPGs may not be correctly positioned anymore relative to the nerves. Again, this may lead to signal loss of the nerves at DWMRN $_{\text {SUSHI }}$ On the other hand, the disappearance of nerve signal at DW-MRN $\mathrm{SUSHI}_{\mathrm{S}}$ does not necessarily have to be a disadvantage. In fact, it may even provide some kind of diagnostic information, analog to the use of fractional anisotropy measurements at DTI [16]. Further exploration with this technique in patients with peripheral nerve pathology (i.e., nerve degeneration, nerve trauma, and nerve tumors) is needed to assess its clinical value.

In this study, any information regarding the applied MR sequence was removed from all images, and images were evaluated in a random order. However, it should be acknowledged that it is not unlikely that the observers could recognize the type of images under evaluation and that this may have introduced some bias.

In conclusion, the proposed SUSHI technique provides an anisotropy-enhanced image, highlighting structures with anisotropic diffusivity, while suppressing structures with a highly isotropic diffusivity. The SUSHI technique allows more selective visualization of the sciatic, common peroneal, and tibial nerves at the level of the knee but is less useful for brachial plexus imaging because signal intensity of the brachial plexus nerves and ganglia can considerably be decreased. 
Conflict of interest statement We declare that we have no conflict of interest.

Open Access This article is distributed under the terms of the Creative Commons Attribution Noncommercial License which permits any noncommercial use, distribution, and reproduction in any medium, provided the original author(s) and source are credited.

\section{References}

1. Maravilla KR, Bowen BC (1998) Imaging of the peripheral nervous system: evaluation of peripheral neuropathy and plexopathy. AJNR Am J Neuroradiol 19:1011-1023

2. Aagaard BD, Maravilla KR, Kliot M (2001) Magnetic resonance neurography: magnetic resonance imaging of peripheral nerves. Neuroimaging Clin N Am 11:131-146

3. Quencer RM (1998) The next frontier in neuroradiology: imaging the peripheral nervous system. AJNR Am J Neuroradiol 19:1001

4. Van Es HW (2001) MRI of the brachial plexus. Eur Radiol $11: 325-336$

5. Freund W, Brinkmann A, Wagner F et al (2007) MR neurography with multiplanar reconstruction of 3D MRI datasets: an anatomical study and clinical applications. Neuroradiology 49:335-341

6. Takahara T, Hendrikse J, Yamashita T et al (2008) Diffusionweighted MR neurography of the brachial plexus: feasibility study. Radiology 249:653-660

7. Yamashita T, Kwee TC, Takahara T (2009) Whole-body magnetic resonance neurography. N Engl J Med 361:538-539
8. Basser PJ, Pajevic S, Pierpaoli C, Duda J, Aldroubi A (2000) In vivo fiber tractography using DT-MRI data. Magn Reson Med 44:625-632

9. Mori S, Kaufmann WE, Davatzikos C et al (2002) Imaging cortical association tracts in the human brain using diffusiontensor-based axonal tracking. Magn Reson Med 47:215223

10. Beaulieu C (2002) The basis of anisotropic water diffusion in the nervous system - a technical review. NMR Biomed 15:435455

11. Haacke EM, Brown RW, Thompson MR, Venkatesan R (1999) Magnetic resonance imaging: physical principles and sequence design, 1st edn. Wiley-Liss, New York, p 632

12. Takahara T, Hendrikse J, Kwee TC et al (2009) Diffusionweighted MR neurography of the sacral plexus with unidirectional motion probing gradients. Eur Radiol 20:1221-1226. doi:10.1007/ s00330-009-1665-2

13. Skorpil M, Engström M, Nordell A (2007) Diffusion-directiondependent imaging: a novel MRI approach for peripheral nerve imaging. Magn Reson Imaging 25:406-411

14. Nonomura Y, Yasumoto M, Yoshimura R et al (2001) Relationship between bone marrow cellularity and apparent diffusion coefficient. J Magn Reson Imaging 13:757-760

15. Raphael DT, McIntee D, Tsuruda JS, Colletti P, Tatevossian R (2005) Frontal slab composite magnetic resonance neurography of the brachial plexus: implications for infraclavicular block approaches. Anesthesiology 103:1218-1224

16. Takagi T, Nakamura M, Yamada M et al (2009) Visualization of peripheral nerve degeneration and regeneration: monitoring with diffusion tensor tractography. Neuroimage 44:884892 